\title{
Erratum: Hyperscaling violating black hole solutions and magneto-thermoelectric DC conductivities in holography [Phys. Rev. D 96, 046015 (2017)]
}

\author{
Xian-Hui Ge, Yu Tian, Shang-Yu Wu, and Shao-Feng Wu
}

(Q) (Received 4 February 2018; published 17 April 2018)

DOI: 10.1103/PhysRevD.97.089901

In Eq. (1), the sign of the kinetic term of the dilaton field should be replaced by a positive sign. So should the sign of the kinetic term in Eq. (2). However, this can lead to states with unbounded negative energy, and instabilities will arise because one of the null energy conditions is violated. We analyze the possible consequences of such a "phantomlike" dilaton field as what follows.

We consider the Einstein-Maxwell-dilaton theory with additional axion fields, which is used for breaking translational invariance in the boundary theory,

$$
S=\int d^{4} x \sqrt{-g}\left[\left(R+V(\phi)+\frac{1}{2} \partial \phi^{2}-\frac{1}{2} Y(\phi) \sum_{i=1}^{2} \partial \chi_{i}^{2}\right)-\frac{1}{4} Z(\phi) F^{2}\right]
$$

where we selected $16 \pi G=g^{2}=L=k_{B}=e=1$, where $L$ is the anti-de Sitter radius, $g^{2}$ is the $3+1$-dimensional gauge coupling constant, and $G$ is Newton's constant.

We choose the following ansatz for the metric, scalar, and gauge field:

$$
d s^{2}=r^{-2 \theta / d}\left(-r^{2 z} f(r) d t^{2}+\frac{d r^{2}}{r^{2} f(r)}+r^{2} d \vec{x}^{2}\right), \quad \phi=\phi(r), \quad F_{i r t} \neq 0 .
$$

In this case, the combining of the $t t$ and $r r$ components of the Einstein equation, one has

$$
R_{t}^{t}-R_{r}^{r}=\frac{1}{2} g^{r r}\left(\partial_{r} \phi\right)^{2} .
$$

Meanwhile, from the Ricci tensor for our ansatz, we obtain

$$
R_{t}^{t}-R_{r}^{r}=-d(1-\theta / d)(z-1-\theta / d) r^{-2 \theta / d} f(r) .
$$

From Eqs. (3) and (4), we obtain

$$
e^{\phi}=r^{\sqrt{-2 d(1-\theta / d)(z-1-\theta / d)}} .
$$

In the special case $d=2, z=1$, and $\theta=1$, we have $\phi=\ln r$.

The full black hole solution is given by

$$
\begin{aligned}
d s^{2} & =-g_{t t} d t^{2}+g_{r r} d r^{2}+g_{x x}\left(d x^{2}+d y^{2}\right) \\
& =r^{-\theta}\left[-r^{2} f(r) d t^{2}+\frac{d r^{2}}{r^{2} f(r)}+r^{2}\left(d x^{2}+d y^{2}\right)\right], \\
f(r) & =1-\frac{m}{r^{2}}-\frac{q^{2} \ln r}{2 r^{2}}-\frac{\beta^{2}}{r}+\frac{B^{2}}{8 r^{4}}, \\
A & =q \ln r d t+\frac{B}{2}(x d y-y d x), \quad e^{\phi}=r, \quad \chi_{i}=\beta \delta_{i a} x^{a}, \\
V(\phi) & =2 r+\mathcal{C} r^{-3}, \quad Z(\phi)=r^{-1}, \quad Y(\phi)=r,
\end{aligned}
$$




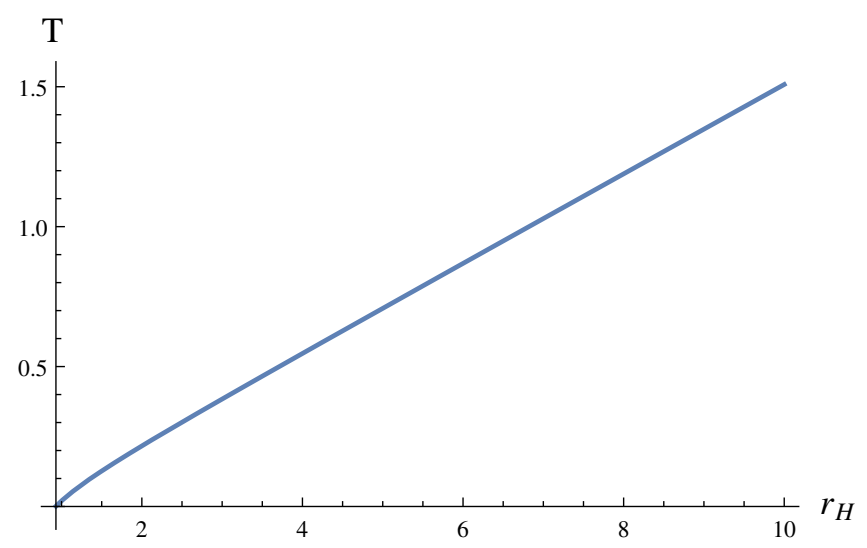

FIG. 1. The Hawking temperature as a function of the event horizon radius.

where $\theta, m, q$, and $B$ are the parameters related to the hyperscaling violation factor, mass, charge, and magnetic field, respectively. Solving the equations of motion, we find that only when the constant $\mathcal{C}$ takes the form $\mathcal{C}(r)=B^{2} / 8$ is the Einstein equation satisfied. It is intriguing to see that the magnetic field $B$ appears in the potential $V(\phi)$.

Now, the problem is that the null energy conditions $(z-1)(d+z-\theta) \geq 0$ and $(d-\theta)[d(z-1)-\theta] \geq 0$ are violated as $d=2, z=1$, and $\theta=1$.

As it was noticed that the violation of the null energy condition may lead to instabilities of the black hole solution, we then check the locally thermodynamical stability of the black hole solution. The Hawking temperature as a function of the event horizon radius reveals that there does not exist an unstable branch of black hole solution as shown in Fig. 1. The specific heat is given by

$$
c_{q, B}=4 \pi^{2} T\left(1+\frac{\beta^{2}+4 \pi T}{\sqrt{4 q_{2}^{2}+\left(\beta^{2}+4 \pi T\right)^{2}}}\right),
$$

which is positive defined.

Indeed, the violation of the null energy condition strongly indicates that the black hole background is unstable. The analysis of the vector and scalar types of perturbations shows that there is no causality violation in the boundary field theory and the sound of velocity is real and positive [1]. However, a thorough study of the black hole instability needs to check the spectrum of quasinormal modes and examine whether or not there are poles crossing to the upper half of the complex frequency plane. In Ref. [2], the instabilities of black holes supported by a phantom scalar field were investigated under spherically symmetric perturbations, and instabilities were found. We would like to perform a thorough study of stability in our black hole background in a future paper [1].

This work was partially supported by NSFC China (Grant No. 11375110); NSFC Grant No. 11475179; the Ministry of Science and Technology (Grant No. MOST 104-2811-M-009-068) and National Center for Theoretical Sciences in Taiwan; and by NSFC China (Grant No. 11275120).

[1] X.H. Ge and Q.-B. Wang (to be published).

[2] K. A. Bronnikov, R. A. Konoplya, and A. Zhidenko, Instabilities of wormholes and regular black holes supported by a phantom scalar field, Phys. Rev. D 86, 024028 (2012). 